\title{
Die Absicherung familialer Sorgearbeit in der Rente. Entwicklungen und Konzepte im internationalen Vergleich
}

Patricia Frericks

\section{Einleitung}

Die Alterssicherung ist ein ganz wesentlicher Teil der sozialen Sicherung. So geht in den meisten Staaten, die Sozialsysteme etabliert haben, der größte Teil der Sozialausgaben in die Alterssicherung, sie betrifft eine recht große Bevölkerungsgruppe, deren Mitglieder, zumindest traditionell, nicht als Erwerbspersonen gelten, und sie stellt dabei als einzige soziale Sicherung eine gesellschaftliche Generalabrechnung des Lebens dar.

In den Diskursen zur Alterssicherung, sowohl den politischen als auch den wissenschaftlichen, steht zumeist die sozialrechtliche Relevanz von Arbeit im Mittelpunkt, das heißt sie fokussieren auf die Absicherung von Erwerbsarbeit in der Rente. In europäischen Wohlfahrtsstaaten variiert allerdings die Interpretation dessen, was bei einer solchen Generalabrechnung des Lebens gesellschaftlich als relevant angesehen wird. Und tatsächlich war Arbeitsmarktpartizipation traditionell in fast keinem Land der alleinige, in vielen nicht einmal der wichtigste Referenzpunkt: Andere bedeutsame Referenzpunkte waren die Familie als Ort der Sorgearbeit und in einigen Wohlfahrtsstaaten der Wohnsitz im Land als Voraussetzung für gesellschaftliche Partizipation.

Auch die Veränderungen der Rente durch zum Teil umfassende Reformen, die in den letzten zwei bis drei Jahrzehnten in allen europäischen Staaten vorgenommen wurden, können mit dem einseitigen Fokus auf der Absicherung von Erwerbsarbeit nicht angemessen erfasst werden. Denn in vielen Wohlfahrtsstaaten wurde die rentenrechtliche Anerkennung von Familie dahingehend geändert, dass nun individuelle Arbeitsmarktpartizipation unterstellt und von Rentenanwartschaften für familiale Sorgearbeit ergänzt wird.

Im deutschen öffentlichen Diskurs werden diese gesellschaftlichen und wohlfahrtsstaatlichen Grundlagen und Entwicklungen oft ignoriert und die Absicherung familialer Sorgearbeit in der gesetzlichen Rentenversicherung als „versicherungsfremde Leistung“ diffamiert. Diese Interpretation ist gerade für einen als konservativ beschriebenen, familienorientierten 
Wohlfahrtsstaat besonders verwunderlich. Schließlich war hier der (männliche) Arbeitnehmer als Familien(haupt)ernäher, also nicht als Individuum, sondern als ein Familienmitglied, sozial- und rentenversichert. Die insbesondere dem Mann und der Frau zugewiesenen Rollen in der Familie wurden über das (Familien-)Einkommen finanziert, und auch für die sozialrechtliche Absicherung war die Familie die wohlfahrtsstaatlich festgeschriebene Einheit. Familie als Ort der Sorgearbeit war also immer Teil der deutschen Sozialversicherungen. Diesen Bezug für Deutschland als versicherungsfremd zu bezeichnen, ist empirisch wie theoretisch falsch.

Dieser Beitrag soll den deutschen Diskurs ein wenig auf die Füße stellen, indem er danach fragt, inwieweit europäische Wohlfahrtsstaaten familiale Sorgearbeit rentenrechtlich absichern, wie sich dieses Ausmaß in den letzten Dekaden verändert hat und welche Konzepte in der Absicherung familialer Sorgearbeit unterschieden werden können. Dabei umfasst familiale Sorgearbeit die von Familienmitgliedern übernommene Erziehung von Kindern und die Pflege bedürftiger Angehöriger. Sie kann, je nach Land und Zeitpunkt, unentgeltlich oder (teil-)vergütet durchgeführt werden.

Zunächst diskutiere ich hierzu den Forschungsstand zum Thema und skizziere das methodische Vorgehen. Anschließend arbeite ich die Differenzen und Entwicklungen der rentenrechtlichen Bedeutung familialer Sorgearbeit im internationalen Vergleich heraus. Bevor der Text mit einem Fazit schließt, kategorisiere ich die in Bezug auf die Absicherung von Sorgearbeit identifizierten Konzepte.

\section{Forschungsstand}

Der Einbezug familialer Sorgearbeit bei der Berechnung sozialer Rechte im Allgemeinen und Renten im Besonderen wurde bislang wenig erforscht. Dabei wissen wir spätestens seit Esping-Andersens breit adaptierter Studie von 1990 (Esping-Andersen 1990), dass etablierte Wohlfahrtsstaaten Sorgearbeit über die Absicherung der Familie und zum Teil über die Absicherung aller im Land wohnenden Personen als zentrale Verteilungskriterien breit abgesichert haben und dass es dabei international und historisch bedeutende Differenzen gibt. In den theoretischen Arbeiten zu Sozialrechten, wie sie vor allem von Marshall (1992) vorgelegt wurden, ist Sorgearbeit selbstverständlicher, dabei aber kaum reflektierter Bestandteil etablierter Sozialrechtskonzepte. Die Familie war für Marshall wie für viele seiner Zeitgenossen selbstverständlich. Auch Adenauer soll im Rahmen der Rentenreform von 1957 gesagt haben „Kinder kriegen die Leute immer!“ Sie 
war einfach da, die Familie, und mit ihr die geleistete Sorgearbeit, geleistet zumeist von der Ehefrau und abgesichert über die Erwerbstätigkeit des Ehemanns. Niemand der männlichen Wortführer des damaligen Rentendiskurses ahnte, dass diese Selbstverständlichkeit keine war.

In der feministischen Wohlfahrtsstaatsforschung wurde die Problematik, dass Frauen über das Familienoberhaupt und über abgeleitete Rechte sozial abgesichert waren, breit diskutiert. Eins der herausragenden Konzepte, das in diesem Kontext entwickelt wurde, ist das der (De-)Familialisierung. Es wurde dazu genutzt, die geschlechtsspezifische Wirkung sozialer Rechte international zu vergleichen. Dieser Vergleich umfasste zum einen das Ausmaß, in dem Wohlfahrtsstaaten Frauen eine finanzielle Unabhängigkeit vom (männlichen) Partner ermöglichen, und zum anderen das Ausmaß, in dem Wohlfahrtsstaaten Familien, insbesondere die weiblichen Familienmitglieder, von familialen Pflege- und Betreuungsarbeiten entlasten (Lister 1994; Leitner 2003). Die Absicherung von Sorgearbeit geht jedoch zum einen über partnerschaftliche Beziehungen weit hinaus und umfasst auch andere Familienmitglieder auf sehr verschiedene Art und Weise (Frericks et al. 2018b). Zudem ist sie komplexer als es der Entlastungsansatz nahelegt, da Sozialrechtsansprüche nicht entlang einer „defamilialisierenden" Logik zugeteilt werden, sondern meist Wahlmöglichkeiten eröffnen, die jeweils zu Sozialrechtsansprüchen führen (Eggers et al. 2019). Daher ist das Konzept der Defamilialisierung für diese Analyse ungeeignet.

Trotz der Aufmerksamkeit, die Sorgearbeit insbesondere bei feministischen Forscherinnen erhält, fehlen in der Forschung bis heute systematische Analysen dazu, inwiefern sie bei der Berechnung von Renten in verschiedenen Ländern einbezogen wurde und wird. Dies liegt wohl auch daran, dass die Methoden und die Datenlage für derlei Fragestellung als schwierig bezeichnet werden müssen. Gemeinhin gilt für Daten, dass nicht, oder nicht systematisch, erfasst wird, was nicht im Fokus steht. Auch fehlt es nach starken kulturellen, sozialen, politischen und wirtschaftlichen Veränderungen an aktualisierten Konzepten zur sozialrechtlichen Relevanz von Sorgearbeit, schließlich wird sie nicht mehr selbstverständlich über einen männlichen Hauptverdiener abgesichert (Frericks et al. 2006 und 2008). Es gibt bislang also keine ausreichende Erforschung der Differenzen und Entwicklungen von sozialpolitischen Ordnungen und Konzepten in Bezug auf die Absicherung familialer Sorgearbeit in der Rente. Ich selber habe in den letzten Jahren zahlreiche empirische, konzeptuelle und methodische Arbeiten vorgelegt, die dazu beitragen können, diese Lücke zu schließen. Und so analysiere ich in diesem Beitrag auf der Basis dieser Vorarbeiten die Absicherung familialer Sorgearbeit in der Rente. 
Dabei arbeite ich die Differenzen und Entwicklungen im internationalen Vergleich heraus und systematisiere die in dieser Analyse identifizierten Konzepte vor dem Hintergrund des deutschen Diskurses.

\section{Theoretische Annahmen zum Zusammenhang zwischen der Absicherung familialer Sorgearbeit und verschiedenen Typen von Wohlfahrtsstaaten}

Welche theoretischen Annahmen liegen nahe, wenn uns die Differenzen in der Absicherung familialer Sorgearbeit im internationalen Vergleich interessieren? Zunächst einmal legt die vergleichende Wohlfahrtsstaatsliteratur nahe, dass sich Wohlfahrtsstaaten in Bezug auf die Absicherung von Sorgearbeit ähnlich unterscheiden wie in Einteilungen von Studien mit anderem Fokus. Grundlegend für diese verschiedenen Studien ist die Wohlfahrtsregimetypologie von Esping-Andersen (1990). Er unterscheidet anhand des Konzepts der Dekommodifikation, das den Fokus auf die Absicherung der Sozialbürger als Arbeiter (sic) legt, drei Regimetypen. Diese unterscheiden sich in ihren Regelungsprinzipien und ihren Umverteilungsmechanismen. So wirkt der egalitär ausgerichtete „sozialdemokratische“ Typ skandinavischer Länder tatsächlich egalisierend, der auf den Markt ausgerichtete „liberale“ Typ anglo-amerikanischer Länder sozialstrukturell dualisierend und der auf traditionelle Kollektive (Familie, Berufe) ausgerichtete „konservativ-korporatistische“ Typ westeuropäischer Länder tatsächlich konservierend. Diese Typologie, die die Ziele wohlfahrtsstaatlicher Ordnung mit ihren Wirkungsweisen verknüpft, wurde um weitere Typen wie dem mediterranen und verschiedenen asiatischen und osteuropäischen ergänzt (eine Übersicht geben Ferragina und Seeleib-Kaiser 2011).

Die Argumente, die angelehnt an diese Regimetypologie in Bezug auf die hier angestrebte Analyse vorgebracht werden können, sind dabei durchaus nachvollziehbar und führen zu Annahmen hinsichtlich der Differenzen in der Altersabsicherung familialer Sorgearbeit, die im empirischen Teil überprüft werden sollen. So würde man für Länder, die dem sozialdemokratischen Wohlfahrtsstaatstypus zugeordnet werden, erwarten, dass sie aufgrund ihrer egalitären Gesellschaftsordnung, die sich in Renten als Wohnsitz-basierte Volksrenten darstellt, keine explizite Absicherung von Sorgearbeit kennen. Tatsächlich sichert ein solches Volksrentensystem weder Erwerbsarbeit noch Sorgearbeit ab oder, anders interpretiert, beide. Zudem hilft der sozialdemokratische Wohlfahrtsstaat bei der Sorgearbeit mit zahlreichen Dienstleistungen, denn Sorgearbeit gilt eher als staatliche denn als familiale Aufgabe. Auch liberale Länder gelten als egalisierend, al- 
lerdings über Marktmechanismen. Staatliche Eingriffe in die Absicherung "familiärer Angelegenheiten" gelten dieser Interpretation nach als systemfremd. Sozialdemokratische und liberale Wohlfahrtsstaaten dürften folglich keine expliziten Anwartschaften für Sorgearbeit in der Rente vorweisen.

Anders stellen sich die Annahmen für konservative Länder dar, die dafür bekannt sind, Familie als den wichtigsten Ort für Sorgearbeit anzusehen. Entsprechend sichern sie Sorgearbeit traditionell über den Familienernährer und die Familienmitgliedschaft $\mathrm{ab}$. Auch mediterrane Länder werden wie die konservativen Wohlfahrtsstaaten zu den familialistischen Wohlfahrtsstaaten gezählt (Naldini 2003). Daher kann auch für sie angenommen werden, dass Sorgearbeit über den Familienernährer und die Familienmitgliedschaft abgesichert ist.

Die wohlfahrtsstaatliche Typisierung der postsozialistischen Länder Ostund Mitteleuropas ist umstritten (Fenger 2007). Während einige Autoren sozialdemokratisch „gemäßigte“ liberale Regime in Mittelosteuropa identifizieren (Szalai 2005), plädieren andere für einen besonderen Bismarck-Typus, der speziell den hybriden Charakter der Visegrád-Staaten betont (Tschechien, Polen, Slowakei, Ungarn) (Kovac 2003). Kollmorgen (2009) zufolge repräsentieren die postsozialistischen Länder mehrere von der ursprünglichen Regimetypologie abweichende Typen. Diesen verschiedenen Studien zufolge kann angenommen werden, dass Länder, die eher als liberal eingestuft werden, wie Estland und Tschechien, Sorgearbeit kaum in der Rente berücksichtigen, während andere, als konservativ geltende Länder, wie Ungarn oder Polen, Sorgearbeit stark über den Familienernährer und die Familienmitgliedschaft absichern.

Diese auf den gängigen Regimetypen basierenden Annahmen wurden bislang jedoch nicht überprüft. Wir wissen also nicht, ob sie der Realität entsprechen. Tatsächlich zeigen einzelne Studien, dass ein von der Dekommodifikation abweichender analytischer Fokus andere als die regimetypenkonformen Ländereinteilungen hervorbringt (Saraceno/Keck 2010, Frericks et al. 2018b). Und da auch die hier angestrebte Analyse der rentenrechtlichen Bedeutung von Sorgearbeit eines anderen Konzeptes als das der Dekommodifizierung oder auch der Defamilialisierung bedarf, würden Ergebnisse mit einer von den Regimetypen abweichenden Ländereinteilung kaum überraschen.

In Bezug auf die Entwicklungen sozialrechtlicher Ordnungen legt die Literatur verschiedene, zum Teil widersprüchliche Annahmen nahe. So wird zum einen davon ausgegangen, dass die zunehmende Bedeutung des adult worker model in europäischen Wohlfahrtsstaaten dazu führt, dass die Relevanz von Sorgearbeit für Sozialrechtsansprüche abnimmt (Lewis/Giullari 
2005, Kvist et al. 2008). Die entsprechende Annahme für die folgende Analyse lautet, dass in Ländern, in denen Sorgearbeit in der Rente abgesichert ist, das Ausmaß dieser Absicherung in den letzten Jahrzehnten abgenommen hat, und in Ländern, in denen Sorgearbeit keine Rolle spielte, sie an Bedeutung nicht gewonnen haben dürfte. Eine andere Lesart geht davon aus, dass der finanzielle Druck, unter dem Wohlfahrtsstaaten stehen, zu einer Abnahme wohlfahrtsstaatlicher „Geschenke“ führe (einen Überblick über die so genannte retrenchment Debatte gibt Starke 2006). Die daraus folgenden Annahmen entsprächen denen der ersten Lesart. Eine weitere Interpretation sieht eher Pfadabhängigkeiten vorherrschen und geht davon aus, dass die Differenzen und damit die Ländereinteilungen mehr oder weniger bestehen bleiben (Esping-Andersen prägte hierfür schon 1990 den Begriff der frozen landscapes). Die entsprechende Annahme wäre, dass sich keine relevanten Entwicklungen erkennen lassen.

Aber auch in Bezug auf die Veränderungen muss man feststellen, dass die sozialrechtliche Bedeutung von Sorgearbeit bislang kaum, und sicher nicht systematisch vergleichend untersucht wurde. Einzelne Studien zur sozialrechtlichen Bedeutung von Sorgearbeit legen nahe, dass sich Wohlfahrtsstaaten in Bezug auf Sorgearbeit und deren Absicherung stark verändert und Sozialrechte für Sorgearbeit ausgebaut haben (Knijn/Kremer 1997, Pfau-Effinger 2004, Frericks et al. 2008). Dies gilt nach ersten Erkenntnissen jedoch nicht für alle Länder oder Regimetypen. Mediterrane Länder beispielsweise gelten insbesondere in Bezug auf Familie als wenig wandlungsfähig, während für Spanien gezeigt wurde, dass es sich in der wohlfahrtsstaatlichen Rahmung von Familie stark modernisiert (Naldini/ Jurado 2013). Für Länder gleichen Regimetyps liegt somit die Annahme nahe, dass sie sich in Bezug auf die Absicherung familialer Sorgearbeit unterschiedlich entwickeln.

Die verschiedenen Annahmen können anhand der folgenden Analyse hervorragend überprüft werden. Der Beitrag wird in einem nächsten Schritt aufzeigen, welche Differenzen und welche Entwicklungen es in der rentenrechtlichen Bedeutung von Sorgearbeit im internationalen Vergleich gibt. Dazu skizziere ich zunächst das methodische Vorgehen für eine solche Analyse.

\section{Methodisches Vorgehen}

In verschiedenen Vorarbeiten habe ich gezeigt, dass man Institutionen in Bezug auf ein bestimmtes Phänomen, wie hier den Einbezug von Sorgearbeit in die Berechnung von Sozialrechten, systematisch analysieren und 
vergleichen kann. Hierzu habe ich mit meinem Forschungsteam die Methode Comparative Messurement of Institutions (CMI) entwickelt, die ich hier nur skizzieren möchte (detailliert in Frericks et al. 2018a). Die Methode arbeitet mit Weber'schen Idealtypen und spannt einen Merkmalsraum auf, in dem die empirische Ausprägung institutioneller Regelungen dargestellt werden kann. Hierzu werden die relevanten Indikatoren, die in der Wohlfahrtsstaatsanalyse zumeist qualitativen Charakters sind, systematisch quantifiziert. Die Methode wurde explizit entwickelt, um Institutionen zu analysieren. Faktisch gewährte Leistungen, die mit anderen Methoden erfasst werden, sind mit dieser wohlfahrtsstaatlichen Gestaltung nicht identisch, da sie von zahlreichen zusätzlichen individuellen und strukturellen Faktoren abhängen.

Um die empirische Ausprägung institutioneller Regelungen zu erfassen, werden die für die Fragestellung relevanten Eigenschaften der Institutionen quantifiziert. Es werden also alle wohlfahrtsstaatlichen Regelungen als Indikatoren identifiziert, die Sorgearbeit bei der Leistungsberechnung einbeziehen. Bei der Rente sind das zusätzliche Ansprüche, die dazu beitragen können, dass man das wohlfahrtsstaatliche Zielsicherungsniveau erreicht, das in Deutschland durch den Eckrentner markiert wird. Hierbei ist zu beachten, dass nur die Daten in Werte umgerechnet werden, die gesichert sind. Da aufgrund des empirisch ungewöhnlichen Fokus die Datenlage zum Teil unsicher ist, weisen die herausgearbeiteten Ausprägungen somit die Mindestausprägung aus.

Die konzeptuelle Unterscheidung zweier wohlfahrtsstaatlich regulierter Sicherungsniveaus, dem Armutsvermeidungsniveau und dem Zielsicherungsniveau, ist empirisch und konzeptuell notwendig: empirisch, weil beide Niveaus verschiedenartig reguliert sind, konzeptuell, weil sie andere Konzepte von Sozialbürgerschaft rahmen. Nur das Zielsicherungsniveau bildet die volle, das heißt nicht die geschlechtsdifferenzierte partielle Sozialbürgerschaft ab (Daly 2011, Lister 1994). Entsprechend kann nur die Analyse der Absicherung von Sorgearbeit auf Zielsicherungsniveau eine geschlechtsspezifische Perspektive auf Sozialrechte vermeiden, wie sie die Unterscheidung in armutsvermeidende Renten für Frauen und angemessene, lebensstandardsichernde Renten für Männer gern vornimmt (s. Frericks 2011). Um die entsprechenden Regelungen auf dem Zielsicherungsniveau zu erfassen, werden die öffentlichen Renten der Länder analysiert sowie in manchen Ländern zusätzlich die obligatorischen Zusatzversicherungen, die sehr verschiedener Art sein können. Solch obligatorische Zusatzversicherungen finden wir in sechs der zehn analysierten Länder: Tschechien, Dänemark, Estland, Schweden, Frankreich und Großbritannien. 
Die Analyse der Differenzen und der Veränderungen wird also für zehn europäische Länder vorgenommen. Die Auswahl soll eine gewisse Varianz garantieren und sowohl die verschiedenen europäischen Wohlfahrtsstaatstypen erfassen als auch Differenzen innerhalb der Typen. Sie umfasst zwei konservativ-korporatistische Länder (Deutschland, Frankreich), zwei sozialdemokratische (Dänemark, Schweden), ein liberales (Großbritannien), zwei mediterrane (Italien, Spanien) und drei postsozialistische (Tschechien, Estland und Ungarn).

Zwei Zeitpunkte werden analysiert: 2018 als für verfügbare Daten aktuellster Moment und 1993 als wohlfahrtsregimetypischer Ausgangspunkt, zu dem noch keine großen Rentenreformen durchgeführt worden waren und die ehemaligen Ostblockstaaten schon mit analysiert werden können.

Zudem werden Ansprüche auf Leistungen für familiale Sorgearbeit nach drei Familiendimensionen unterschieden, um die Empirie sauber und detailliert erfassen zu können: Sorgearbeit für die/den Partner/in, für das/die Kind/er und für weitere Familienangehörige. Relativierungen familialer Sorgearbeit wie in Regelungen, die nur für verheiratete Partner oder nur für im eigenen Haushalt lebende Kinder oder Verwandte gelten, werden systematisch gewichtet. Einbezogen werden die expliziten Regelungen zur Absicherung von Sorgearbeit (Pflege) sowie auf der Kinderdimension auch die Absicherung über den Status als Eltern (indirekte Adressierung von Sorgearbeit). Andere Leistungen, die auf einem Familienstatus wie dem der Ehe beruhen, werden nicht mit aufgenommen, um explizit nur die Absicherung von Sorgearbeit zu erfassen. Regelungen für die Pflege behinderter Kinder werden hier nicht mit einbezogen, da diese besonderen Pflegebedarfe im internationalen Vergleich über sehr verschiedenartige familienpolitische und sozialpolitische Institutionen geregelt und finanziert werden. Aus diesem Grund ergibt sich für die Sorgearbeit für Kinder ein oft niedrigerer Wert als der für die anderen beiden analysierten Familiendimensionen. Dieser niedrigere Wert erklärt sich also aus den hier nicht systematisch einbeziehbaren Sonderregelungen für pflegebedürftige Kinder, die von den generellen Regelungen zur Absicherung familialer Pflege in vielen Ländern deutlich abweichen. Als Datengrundlage dienen internationale Datenbanken zu sozialrechtlichen Regelungen wie MISSOC und ISSA, nationale Regelungsdaten und, wo hilfreich, einschlägige Studien.

Die Werte, die mittels CMI ermittelt werden, können bei jeder Familiendimension idealtypisch zwischen 0 (keinerlei Absicherung von Sorgearbeit) und 1 (volle Rente aufgrund von Sorgearbeit) liegen und addieren sich zu einem Gesamtwert von maximal 3 (alle drei analysierten Familiendimensionen zusammengenommen). Um den Rahmen nicht zu sprengen, werden nur diese Gesamtwerte diskutiert. Die Absicherung familialer Sor- 
gearbeit ist in keinem Land das zentrale Kriterium des Rentenaufbaus. Dieses zentrale Kriterium ist zum einen die Arbeitsmarktpartizipation und in einigen Ländern der Wohnsitz. Die hier ermittelten Werte sind also vor dem Hintergrund des im Land dominierenden Kriteriums zur Rentenberechnung zu sehen.

\section{Ergebnisse zu den Differenzen und Entwicklungen der sozialrechtlichen Bedeutung familialer Sorgearbeit im internationalen Vergleich}

Hinsichtlich der Differenzen der sozialrechtlichen Bedeutung familialer Sorgearbeit im internationalen Vergleich zeigt sich für das Jahr 2018, dass alle zehn analysierten Länder Sorgearbeit in die Berechnung der Alterssicherung einbeziehen. Dabei gibt es zum Teil deutliche Differenzen zwischen den Ländern und den Familiendimensionen (eine Übersicht aller Werte bietet Tabelle 1). Der Ländervergleich zeigt, dass Sorgearbeit zu einem unterschiedlichen Grad Teil der gegenwärtigen Rentenberechnungen ist. 2018 weisen Tschechien und Großbritannien die höchste Absicherung familialer Sorgearbeit in der Rente auf (zwischen 0,7 und 0,8). Eine zweite Ländergruppe folgt mit Gesamtwerten zwischen 0,4 und 0,5 und umfasst Deutschland, Frankreich und Estland. Mit Dänemark und Ungarn zeigt eine dritte Gruppe Werte zwischen 0,2 und 0,3. Schweden und Spanien weisen Werte zwischen 0,1 und 0,2 auf, und Italien den niedrigsten Wert von 0,05 .

Diese Gruppen zeigen, dass sich die Länder in der Absicherung familialer Sorgearbeit in der Rente anders gruppieren als in gängigen Regimetypologien. Zudem entsprechen sie den auf diesen Typologien basierenden Annahmen nicht, denn sowohl (postsozialistisch) liberale als auch sozialdemokratische Länder sichern familiale Sorgearbeit zu einem zum Teil sehr hohen Grad explizit ab. Dabei ist dieser Grad bei den als liberal klassifizierten Ländern deutlich höher als bei den konservativ-korporatistischen und postsozialistisch-konservativen Ländern. Mediterrane Länder wiederum sichern familiale Sorgearbeit am wenigsten ab.

Die Veränderungen, die in der Absicherung von Sorgearbeit in den Analyseländern seit Anfang der 1990er Jahre vorgenommen wurden, lassen sich wie folgt zusammenfassen. Die Gruppen, die sich für das Jahr 1993 herausbilden, stimmen mit denen, die für das Jahr 2018 identifiziert wurden, in keiner Weise überein. So gibt es 1993 nur ein Land mit Werten zwischen 0,7 und 0,8: Tschechien. Zu der Gruppe der Länder mit Werten zwischen 0,4 und 0,5 gehörten Großbritannien und Ungarn, das heißt nur Länder, die 2018 nicht mehr zu dieser Gruppe gehören werden und kei- 
nes, dass 2018 dazugehören wird. Und zu der Gruppe der Länder mit Werten zwischen 0,1 und 0,2 gehörten Deutschland, Frankreich, Estland und Schweden. Auch hier werden 2018 drei der vier Länder nicht mehr zu dieser Gruppe gehören. Gruppen, die an Einteilungen der Regimetypen denken lassen, gibt es auch 1993 nicht. Und die Entwicklungen unterscheiden sich. Sie variieren zwischen deutlich gestiegenen Gesamtwerten in Deutschland, Estland, Frankreich und Großbritannien, einer eher marginalen Steigerung in Dänemark, Italien und Tschechien, und einem deutlich reduzierten Gesamtwert in Ungarn. Dabei zeigt sich keine Korrelation zwischen dem Wert von 1993 und dem Grad der Veränderung. Deutlich ist, dass alle Untersuchungsländer außer Ungarn die Absicherung der Sorgearbeit in der Rente erhöht haben.

Tabelle 1: Die Absicherung von Sorgearbeit in Europa 1993 und 2018

\begin{tabular}{lrrrrrrrrr}
\hline & \multicolumn{4}{c}{1993} & & \multicolumn{2}{c}{2018} & & Differenz \\
& Partner & Kinder & Weitere & Gesamt & Partner & Kinder & Weitere & Gesamt & Gesamt \\
\hline CZ & 0,3 & 0,16 & 0,3 & 0,76 & 0,3 & 0,19 & 0,3 & 0,79 & $+0,03$ \\
D & 0,01 & 0,1 & 0,01 & 0,12 & 0,16 & 0,18 & 0,16 & 0,50 & $+0,38$ \\
DK & 0,11 & 0,01 & 0,11 & 0,23 & 0,11 & 0,02 & 0,11 & 0,24 & $+0,01$ \\
I & 0 & 0 & 0 & 0 & 0 & 0,05 & 0 & 0,05 & $+0,05$ \\
HU & 0,22 & 0,1 & 0,22 & 0,45 & 0,06 & 0,09 & 0,06 & 0,21 & $-0,24$ \\
E & 0 & 0,03 & 0 & 0,03 & 0,02 & 0,09 & 0,02 & 0,13 & $+0,1$ \\
F & 0 & 0,17 & 0 & 0,17 & 0,14 & 0,17 & 0,14 & 0,45 & $+0,28$ \\
EE & 0 & 0,14 & 0 & 0,14 & 0,18 & 0,12 & 0,18 & 0,48 & $+0,34$ \\
UK & 0,17 & 0,12 & 0,17 & 0,46 & 0,3 & 0,17 & 0,3 & 0,77 & $+0,31$ \\
S & 0 & 0,1 & 0 & 0,1 & 0 & 0,18 & 0 & 0,18 & $+0,08$
\end{tabular}

Die dargestellten Veränderungen zeigen deutlich, dass die sozialrechtliche Relevanz von Sorgearbeit mit dem adult worker model nicht abnimmt, sondern in den meisten Ländern Sorgearbeit stärker abgesichert wurde. Der finanzielle Druck, unter dem Wohlfahrtsstaaten stehen, wirkt sich auf die Absicherung von Sorgearbeit folglich nicht abnehmend aus. Dabei unterscheidet sich der Grad der Veränderung nicht systematisch nach Ländern des gleichen Regimetyps. Und auch eine Pfadabhängigkeit lässt sich für die meisten Länder nicht erkennen: Die Hälfte der analysieren Länder nimmt sehr deutliche Änderungen in der Absicherung von Sorgearbeit in der Rente vor.

\section{Diskussion der Ergebnisse}

Die hier analysierten Länder unterscheiden sich in ihren Rentensystemen zum Teil stark voneinander. Ihre Differenzen und Entwicklungen folgen 
unterschiedlichen Konzepten von Renten. In steuerfinanzierten Renten („Volksrenten“), wie es sie in Dänemark und Schweden gibt, ist die Absicherung von Sorgearbeit selbstverständlicher und impliziter Bestandteil der Renten, die ehedem das Zielsicherungsniveau sicherten. Durch die Rentenreformen auf Zielsicherungsniveau, die zusätzliche Institutionen begründeten, also die Zusatzrente ATP in Dänemark und Schweden, kam zu dem zentralen Kriterium des Wohnsitzes (Volksrente) das der Arbeitsmarktpartizipation (ATP) hinzu (Frericks et al. 2006). Sorgearbeit war damit nur noch zum Teil, aber nicht mehr auf Zielsicherungsniveau abgesichert, da in der nun notwendigen ATP die Absicherung von Sorgearbeit fehlt. Zum Teil passten diese Länder ihre in der Logik des Rentenaufbaus grundsätzlich veränderten Systeme mittels gesonderter Regelungen für Sorgearbeit an. So wurde in Dänemark die Möglichkeit eingeführt, dass Pflegende eines Familienmitglieds hierfür als Arbeitnehmer/in der Gemeinde eingestellt werden und so die volle soziale Sicherung auch in den notwendigen Zusatzsystemen aufbauen (Frericks et al. 2014). Eine vergleichbare Regelung zur Absicherung von Sorgearbeit in Bezug auf Kinder gibt es in den genannten Ländern jedoch weiterhin nicht.

Das Rentensystem in Großbritannien mit seinen zwei Grundstöcken, der Basic State Pension (BSP) und der Additional State Pension (Anfang der 1990er Jahre SERPS; später State Second Pension (S2P)), folgt einer anderen Logik. Anders als regimetypologische Annahmen erwarten lassen, umfassen Renten hier starke Familienkomponenten. Dies gilt auch für die postsozialistischen Länder, die gemeinhin als liberal klassifiziert werden. Dass die starken Familienkomponenten auf dem Zielsicherungsniveau zu finden sind und nicht nur, wie für liberale Länder eventuell erwartet, auf dem Armutsvermeidungsniveau oder eher versteckt in steuerlichen Regelungen, ist ein besonders interessantes Ergebnis dieser Arbeit.

In beitragsfinanzierten Sozialversicherungsrenten wie dem in Deutschland wiederum werden Ansprüche über Beiträge erworben. Arbeitnehmer/ innen und Arbeitgeber/innen führen im Normalfall paritätisch die Beiträge entsprechend der Einkommenshöhe ab, zumindest bei mittleren Einkommen. In verschiedenen Diskursen, und gerade auch im aktuellen deutschen Diskurs, wird dabei suggeriert, dass die Rente ein aufgeschobenes Gehalt sei (deferred wage), das beitragsäquivalent ausgezahlt werde (Friot 2004). Die Ressourcen des Rentensystems gehören nach dieser Lesart also den Arbeitnehmern, und wenn aus dem Rentensystem etwas für Sorgearbeit gezahlt wird, werde diese „versicherungsfremde Leistung“ aus dem Eigentum der Arbeitnehmer (sic) gezahlt. Diese sehr vereinfachte Rechnung ist aus zwei Gründen falsch. Zum einen werden alle Äquivalenzsysteme in größerem Umfang mit Steuermitteln ko-finanziert. Zum zweiten sind die 
betroffenen Sozialversicherungssysteme traditionell auf Familie als Versicherungseinheit ausgerichtet und umfassen damit immer auch die familiale Sorgearbeit. Für konservative Wohlfahrtsstaaten heißt das, dass die Absicherung von Sorgearbeit immer wesentlicher Bestandteil des Rentensystems und des Sozialversicherungskonzeptes war. Sie wird jetzt nur anders in die Rentenberechnung einbezogen (Frericks 2014), denn die Familie als Sozialrechtseinheit mit der impliziten Absicherung familialer Sorgearbeit weicht zum Teil der expliziten Absicherung familialer Sorgearbeit als eigene Sozialrechtsbegründung.

\section{Kategorisierung der Konzepte}

In diesem Teil möchte ich eine Übersicht über die in der Analyse identifizierten Instrumente bzw. Konzepte der expliziten Absicherung familialer Sorgearbeit aufführen (siehe Tabelle 2). Die Aufbereitung aller Indikatoren hat ergeben, dass grundsätzlich drei verschiedene Ansätze zur Absicherung der Sorgearbeit unterschieden werden können. Dabei folgen sie, wie ich andernorts gezeigt habe (Frericks 2015), jeweils anderen Begründungen. Die drei Ansätze umfassen zum einen additive Ansprüche, die zu den Ansprüchen aus Arbeitsmarktpartizipation hinzukommen, zum zweiten Ansprüche, die Beiträge aus der ausfallenden Arbeitsmarktpartizipation ersetzen, und zum dritten keine Ansprüche auf die Absicherung familialer Sorgearbeit.

Es zeigt sich, dass der dritte Ansatz, also keine Rentenansprüche aufgrund von Sorgearbeit, empirisch in keinem Wohlfahrtsstaat zu finden ist. Alle analysierten Wohlfahrtsstaaten haben Ansprüche für familiale Sorgearbeit in ihren Rentensystemen verankert. Zudem ist deutlich, dass der erste, additive Ansatz vornehmlich auf Kindererziehung ausgerichtet ist und sich in den verschiedenen Ländern vom zweiten, ersetzenden Ansatz hierhin verschoben hat und zudem erweitert wurde. Der zweite Ansatz, der Sorgearbeit und deren Absicherung unter dem Gesichtspunkt der (teil-)unterbrochenen Arbeitsmarktpartizipation konzipiert, wurde insbesondere zur Absicherung familialer Pflegetätigkeiten eingeführt oder gestärkt. 
Tabelle 2: Absicherung von Sorgearbeit in beitragsfinanzierten Rentensystemen (auf Zielsicherungsniveau)

\begin{tabular}{|c|c|c|}
\hline Instrument / Konzept & Begründung & $\begin{array}{l}\text { Empirische Beispie- } \\
\text { le }\end{array}$ \\
\hline $\begin{array}{l}\text { Zusätzliche Ansprüche, die } \\
\text { zu denen aus gleichzeiti- } \\
\text { ger Arbeitsmarktpartizi- } \\
\text { pation hinzukommen }\end{array}$ & $\begin{array}{l}\text { Pflege und Kindererzie- } \\
\text { hung als gesellschaftli- } \\
\text { cher Beitrag zur Erfül- } \\
\text { lung des Generationen- } \\
\text { vertrages }\end{array}$ & $\begin{array}{l}\text { Tschechien, } \\
\text { Deutschland, Frank- } \\
\text { reich für Kinder }\end{array}$ \\
\hline $\begin{array}{l}\text { Ansprüche, die ausfallende } \\
\text { Beiträge aus der Arbeits- } \\
\text { marktpartizipation erset- } \\
\text { zen }\end{array}$ & $\begin{array}{l}\text { Reduktion der sozial- } \\
\text { rechtlichen Nachteile der } \\
\text { aufgrund familialer Sor- } \\
\text { gearbeit unterbrochenen } \\
\text { oder reduzierten Arbeits- } \\
\text { marktpartizipation }\end{array}$ & $\begin{array}{l}\text { Estland, Frankreich } \\
\text { für Kindererzie- } \\
\text { hung bis } 2004 \\
\text { (später additiv), } \\
\text { Deutschland für } \\
\text { Pflege }\end{array}$ \\
\hline $\begin{array}{l}\text { Keine Ansprüche aufgrund } \\
\text { von Sorgearbeit }\end{array}$ & $\begin{array}{l}\text { „versicherungsfremd“, } \\
\text { Privatversicherungslogik }\end{array}$ & nirgends \\
\hline
\end{tabular}

Die Differenzen zwischen dem ersten, additiven Ansatz und dem zweiten, ersetzenden konnten in der Analyse durch systematische Gewichtungen einbezogen werden und spiegeln sich in den Werten wider. Der dritte Ansatz wurde empirisch nirgends gefunden; tatsächlich widerspricht er der Konzeption und Logik aller Sozialversicherungen (Barr 2004, Hyde et al. 2003). Und nicht nur das, nicht einmal in neu eingeführten marktbasierten Ausgleichsrenten wie der Riesterrente in Deutschland wurde diese Privatversicherungslogik angewandt (vgl. Frericks 2015, Frericks/Höppner 2019).

Abschließend sei gesagt, dass der deutsche Vorstoß, ein neues wohlfahrtsstaatliches Sicherungsniveau, das der so genannten „Lebensleistung“, zu etablieren, letztlich nur eine zweite Form armutsvermeidender Renten darstellt. Die Einführung der Grundrente, also einer auf Armutsvermeidung ausgerichteten Rente für die, die in ihrem Leben gearbeitet und zugleich für Familienmitglieder gesorgt haben, würde die Alterssicherung auf Armutsvermeidungsniveau lediglich aufgespalten in Renten für die, die das Wohlwollen der Gemeinschaft etwas mehr verdienen und die, die es nicht verdienen (zum Diskurs der „deservingness“, siehe Van Oorschot 2006). Sie würde die bisherige Aufteilung der Renten in angemessene Renten für Männer und armutsvermeidende Renten für Frauen weiter zementieren. 


\section{Fazit}

Die Analyse der Absicherung familialer Sorgearbeit in der Rente hat gezeigt, dass Sorgearbeit in Europa ein wichtiges Kriterium in der Berechnung der Renten war und ist. Dabei wurden nur Regelungen zur expliziten Absicherung von Sorgearbeit analysiert und die impliziten, wie sie über ein Volksrentensystem in sozialdemokratischen Ländern oder einen Ehestatus in konservativen Ländern vorherrschen, außen vor gelassen. Alle analysierten Wohlfahrtsstaaten weisen explizite Rentenleistungen für familiale Sorgearbeit auf. Dabei unterscheiden sie sich im Ausmaß der Leistungen, und sie sichern Sorgearbeit für verschiedene Familienmitglieder unterschiedlich $\mathrm{ab}$.

Die auf der Regimetypologie basierenden forschungsleitenden Annahmen wurden ganz oder teilweise widerlegt. Für das Jahr 2018 zeigen die Ergebnisse, dass Sorgearbeit in den sozialdemokratischen Wohlfahrtsstaaten Dänemark und Schweden stärker (explizit) abgesichert und anerkannt ist, als das gängige Verständnis ihrer Sozialsysteme es nahegelegt. Die Wohlfahrtsstaaten Großbritannien und Tschechien wiederum sind nicht so individual-liberalisiert wie aus der Wohlfahrtsstaatliteratur geschlossen werden könnte, im Gegenteil weisen sie die am stärksten ausgeprägte Absicherung familialer Sorgearbeit auf. Konservative Länder hingegen liegen in der Absicherung von Sorgearbeit eher im Mittelfeld, und mediterrane Wohlfahrtsstaaten sichern Sorgearbeit kaum ab. Die fehlende Absicherung von Sorgearbeit in der italienischen Rente bestätigt die früheren Analysen von Saraceno (z.B. Saraceno/Keck 2010), denen zufolge Italiens Wohlfahrtsstaat familialisierend wirkt, indem er nichts regelt (familialisation by default). Es wurde auch die Annahme bestätigt, dass ein andersgearteter Fokus eine Ländereinteilung erwarten lässt, die von den Regimetypen zum Teil abweicht. Denn tatsächlich ist der gegenwärtige Grad der Absicherung familialer Sorgearbeit in Deutschland, Frankreich, aber auch Estland vergleichbar, und so unterschiedliche Wohlfahrtsstaaten wie Schweden, Spanien und Ungarn weisen vergleichbare Werte auf.

Auch die Entwicklung der Absicherung familialer Sorgearbeit in den letzten 35 Jahren unterscheidet sich und variiert zwischen kleinen Zunahmen in Ländern mit zuvor niedrigem wie Ländern mit zuvor hohem Absicherungsniveau der Sorgearbeit und großen Zunahmen in Ländern mit zum Teil sehr verschiedenen Ausgangswerten. Nur in Ungarn nahm die Absicherung von Sorgearbeit (stark) ab, eine Entwicklung, die als Refamilialisierung in traditioneller Hinsicht interpretiert werden kann. Kaum Veränderungen in dem Ausmaß zu der Sorgearbeit abgesichert ist, sehen wir in sehr verschiedenen Ländern wie Tschechien, das einen hohen Wert 
aufweist, Dänemark, das einen mittleren Wert aufweist und Italien, das Sorgearbeit kaum absichert. Auch Spanien änderte sich weniger stark als neuere Studien nahelegen. Eine starke Zunahme der Absicherung von Sorgearbeit hingegen zeigt sich in Deutschland, Estland, Frankreich und Großbritannien. Eine regimetypische Systematik in der Entwicklung der Absicherung familialer Sorgearbeit lässt sich somit nicht erkennen.

Auch andere Annahmen zur Entwicklung der Absicherung von Sorgearbeit wurden weitgehend widerlegt: So nimmt die sozialrechtliche Relevanz von Sorgearbeit mit dem adult worker model nicht ab, sondern in den meisten Ländern zu. Ebenso wenig bewirkt der finanzielle Druck, unter dem Wohlfahrtsstaaten stehen, einen Rückbau dieser Elemente. Und auch eine Pfadabhängigkeit ist für die meisten Länder nicht auszumachen: Die Hälfte der analysierten Länder nimmt sehr deutliche Änderungen in der Absicherung von Sorgearbeit in der Rente vor.

Diese Ergebnisse sind sowohl für die Wohlfahrtsstaats- und Rentenforschung als auch für den öffentlichen Diskurs von großer Bedeutung. So sollten bei Analysen, die nicht ausschließlich oder vornehmlich die Absicherung von Erwerbsarbeit untersuchen, regimeuntypische Ergebnisse durchaus erwartet werden. Und tatsächlich ist die Absicherung von Sorgearbeit sehr komplex und kann mit den gängigen Konzepten nicht erfasst werden. Dies gilt auch für die Entwicklung wohlfahrtsstaatlicher Absicherungslogiken. Abgesichert wird in gegenwärtigen europäischen Wohlfahrtsstaaten verstärkt die Tätigkeit, weniger der Status (vgl. Frericks 2013). Sorgearbeit wird also neben Erwerbsarbeit zunehmend als gesellschaftlich explizit wertgeschätzte Tätigkeit abgesichert.

Zudem konnte gezeigt werden, dass der öffentliche Diskurs, der mit "Versicherungsfremde“ argumentiert, den empirischen und konzeptuellen Grundlagen europäischer Wohlfahrtsstaaten widerspricht. Wenn also Wohlfahrtsstaaten und soziale Sicherungssysteme "modernisiert" werden, um sich den gegenwärtigen Gesellschaften neu anzupassen, sollten „alte“ Einsichten, die weiterhin Gültigkeit haben, angemessen berücksichtigt werden. Familiale Sorgearbeit als gesellschaftliche Größe ist dabei in wohlfahrtsstaatlichen Reformen in ganz Europa präsent. Der Diskurs hingegen, insbesondere der deutsche, ist stark geprägt von einem gesellschaftstheoretisch wie empirisch verfälschend vereinfachten Blick auf wohlfahrtsstaatliche Ordnungen (ausführlich hierzu: Frericks/Maier 2012). Es wäre nur schwerlich nachvollziehbar, wenn in einer Zeit, in der sehr verschiedene gesellschaftliche Akteure laut darüber nachdenken, wie Wohlstand und Wertschöpfung neu und sinnvoll erfasst werden können, der theoretisch und empirisch etablierte Stellenwert von Sorgearbeit vernachlässigt würde. 


\section{Literatur}

Barr, N. (2004): Economics of the Welfare State, Oxford

Daly, M. (2011): What Adult Worker Model? A Critical Look at Recent Social Policy Reform in Europe from a Gender and Family Perspective, in: Social Politics 18 (1), S. 1-23

Eggers, T./Grages, C./Pfau-Effinger, B. (2019): Self-responsibility of the 'active social citizen': Different policy concepts about 'active citizenship' in different types of welfare states, in: Frericks, P./Höppner, J. (Hrsg.): The Turn Toward Self-Responsibility in Current Societies: Differences, Challenges and Perspectives, American Behavioral Scientist Special Issue 63 (1), S. 43-64

Esping-Andersen, G. (1990): The three worlds of welfare capitalism, Cambridge

Fenger, M. (2007): Welfare regimes in central and eastern Europe: Incorporating post-communist countries in a welfare regime typology, in: Contemporary Issues and Ideas in Social Sciences 3 (2), S. 1-30

Ferragina, E./Seeleib-Kaiser, M. (2011): Welfare regime debate: past, present, futures?, in: Policy \& Politics 39 (4), S. 583-603

Frericks, P. (2011): Angemessene und nachhaltige Renten für alle? Die geschlechtsspezifische Wirkung des deutschen Rentensystems, in: Vierteljahrshefte zur Wirtschaftsforschung (DIW), Special Issue: Die Zukunft der Alterssicherung 80 (2), S. 119-132

Frericks, P. (2013): Strengthening Market Principles in Welfare Institutions: How Hybrid Pension Systems Impact on Social-risk Spreading, in: Journal of Social Policy 42 (4), 665-683

Frericks, P. (2014): Unifying self-responsibility and solidarity in social security institutions: The circular logic of welfare-state reforms in Europe, in: European Societies 16 (4), S. 522-542

Frericks, P. (2015): Culture matters: Die Finanzialisierung der Alterssicherung. Ein Ländervergleich, in: Heuer, J.-O./Schraten, J. (Hrsg.): Finanzialisierung des Alltags als Herausforderung für den Sozialstaat, Schwerpunktheft der Zeitschrift für Sozialreform 61 (3), S. 265-289

Frericks, P./Maier, R. (2012): European Capitalist Welfare Societies. The Challenge of Sustainability, London

Frericks, P./Höppner, J. (2019): Does the marketisation of pensions lead to individualisation? An examination of family-related pension entitlements, in: Policy and Politics 47 (4), S. 579-597

Frericks, P./Maier, R./De Graaf, W. (2006), Shifting the Pension Mix: Consequences for Dutch and Danish Women, in: Social Policy and Administration 40 (5), S. 475-492

Frericks, P./Maier, R./De Graaf, W. (2008): Male norms and female adjustments. The influence of care credits on gender pension gaps in France and Germany, in: European Societies 10 (1), S. 97-119 
Frericks, P./Jensen, P.H./Pfau-Effinger, B. (2014): Social rights and employment rights related to family care: Family care regimes in Europe, in: Journal of Aging Studies 29, S. 66-77

Frericks, P./Höppner, J./Och, R. (2018a): The Difficulty of Measuring Institutions: A Methodological Approach to the Comparative Analysis of Institutions, in: Social Indicators Research 137 (3), S. 847-865

Frericks, P./Höppner, J./Och, R. (2018b): Individualisierung der Sozialbürgerschaft? Entwicklungen und Widersprüche in Europa, in: Berliner Journal für Soziologie 28 (3), S. 427-456

Friot, B. (2004): Resource regime reforms and worker status, in Clasquin, B./ Moncel, N./Harvey, M./Friot, B. (Hrsg.): Wage and Welfare. New Perspectives on employment and social rights in Europe, Brüssel S. 75-99

Hyde, M./Dixon, J./Drover, G. (2003): Welfare Retrenchment or Collective Responsibility? The Privatisation of Public Pensions in Western Europe, in: Social Policy \& Society 2 (3) S. 189-197

Knijn, T./Kremer, M. (1997): Gender and the caring dimension of welfare states: toward inclusive citizenship, Social Politics Special Issue 4 (3), S. 328-361

Kollmorgen, R. (2009): Postsozialistische Wohlfahrtsregime in Europa - Teil der „Drei Welten“ oder eigener Typus? Ein empirisch gestützter Rekonzeptualisierungsversuch, in: Pfau-Effinger, B./Sakač-Magdalenić, S./Wolf, C. (Hrsg.): International vergleichende Sozialforschung. Ansätze und Messkonzepte unter den Bedingungen der Globalisierung, Wiesbaden, S. 65-92

Kovacs, J. (2003): A Cusion that Suffocates? Transforming the „Communist Welfare State“ in East-Central Europe, in: Kovacs (Hrsg.): Small Transformations. The Politics of Welfare Reform - East and West, Münster u.a., S. XIII-XLII

Kvist, J./Pedersen, L./Köhler, P.A. (2008): Making all persons work: modern Danish labour market policies, in: Eichhorst, W./Kaufmann O./Konle-Seidl, R. (Hrsg.): Bringing the jobless into work? Experiences with activation schemes in Europe and the US, Berlin/London, S. 222-256

Leitner, S. (2003): Varieties of familialism: The caring function of the family in comparative perspective, in: European Societies 5 (4), S. 353-375

Lewis, J./Giullari, S. (2005): The adult worker model family, gender equality and care: the search for new policy principles and the possibilities and problems of a capabilities approach, in: Economy and Society 34 (1), S. 76-104

Lister, R. (1994): 'She has other duties' -Women, citizenship and social security, in: Baldwin, S./Falkingham, J. (Hrsg.): Social security and social change. New challenges to the Beveridge model, New York/London, S. 31-44

Marshall, T.H. (1992): Bürgerrechte und soziale Klassen. Zur Soziologie des Wohlfahrtsstaates. Hrsg. v. E. Rieger, Frankfurt a. M.

Naldini, M. (2003): The Family in the Mediterranean Welfare State, London

Naldini, M./Jurado, T. (2013): Family and Welfare State Reorientation in Spain and Inertia in Italy from a European Perspective, in: Population Review 52 (1), S. 43-61 
Pfau-Effinger, B. (2004): Historical paths of the male breadwinner family model explanation for cross-national differences, in: British Journal of Sociology 55 (3), S. $177-199$

Saraceno, C./Keck, W. (2010): Can We Identify Intergenerational Policy Regimes in Europe?, in: European Societies 12 (5), S. 675-696

Starke, P. (2006): The politics of welfare state retrenchment: A literature review, in: Social Policy \& Administration 40 (1), S. 104-120

Szalai, E. (2005): Socialism. An analysis of its Past and Future, Budapest/New York

Van Oorschot, W. (2006): Making the difference in social Europe: Deservingness perceptions among citizens of European welfare states, in: Journal of European Social Policy 16 (1), S. 23-42 